\title{
Study on System Architecture Design of University Students Quality Evaluation
}

\author{
He Yongqiang ${ }^{\mathrm{a}}$, Wang Jiaxin ${ }^{\mathrm{b}}$ \\ ${ }^{a, b}$ Henan Institute of Engineering, Zhengzhou, China
}

\begin{abstract}
In order to improve the comprehensive qualities of students in all respects, fully reflect and evaluate the achievements of students, inspire students to improve themselves unceasingly, encourage them to strive to be the best, develop their personalities and strive for truth and innovation, as well as achieve the institutionalization, standardization and scientificalness of students management work gradually, meanwhile, along with the development of university construction and students management, maintenance and management of students' data information has been exceptionally heavy, so it cries out for a set of reasonable and convenient students quality evaluation system. Students' quality evaluation system is designed to make better use of computer technology in managing and inquiring about total performance of students' qualities. Through analysis of the objective of system construction, combining design principle of the system, this thesis analyses and designs the quality evaluation system architecture from the perspectives of architecture, function module and security model.
\end{abstract}

Index Terms: quality evaluation; system architecture; construction objective; function module; security model

(C) 2012 Published by MECS Publisher. Selection and/or peer review under responsibility of the Research Association of Modern Education and Computer Science

\section{Introduction}

No matter what kind of mode adopted in carrying out quality education, there must be corresponding evaluation system for students' comprehensive quality level. Evaluation not only checks out comprehensive education level but also promotes the implementation and improvement of it. The result of evaluation can direct the personalization education; students can make personal development planning according to evaluation results; teacher can teach students according to their aptitude on the basis of evaluation results. With the development of university construction and student management, number of enrolled students increases steadily, campus area becomes larger and lager, work of maintaining and managing students' data information becomes exceptionally heavy, the traditional evaluation and test mode is becoming less and less suitable for modern management and education. Managing the enormous and complex students' information by computer is not only convenient for

\footnotetext{
The key scientific and technological project of the Education Department of Henan Province.

* Corresponding author.

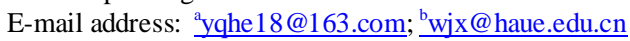


query, correction and statistic but also effective and rapid which can satisfy requirements of modern management.

\section{Construction Objective of the System}

The overall objective of evaluation system is to manage the students' integrated quality evaluation information in a more effective way through implementation of this system. By widely taking students' and employing units' advice, update the contents of courses in universities and improve teaching and management level; through accurate data acquisition and processing, provide accurate foundation for education and management of students, improve the work accuracy and work efficiency to save resources. So the construction objectives of system are:

1) System possesses higher reliability and usability: It should run without failure for extended period of time. The core devices network of it and database server should be equipped with backup server; copy and backup data in database regularly. If there is any unexpected failure to server, the whole system should be able to automatically switch to backup server for continuous work to avoid loss of data.

2) System is easy to manage and maintain: It should possess simple and convenient system management tools such as tools for user authority management, each functional management, system operation parameter management, run-log management, integrated network management and system monitoring, debug and diagnostics.

3) System is easy to learn and use: The system is often under concurrency operation by many people, or one person operates many subsystems. The users' computer skill is different, so the system should have a simple and flexible friendly human-computer interface.

4) System should have good scalability and openness: It can be built up in different systems with better client-side system scalability; the system with better openness can be interlinked with other systems.

5) Favorable cost performance: Under the precondition of meeting the demand of application function, system performance and guaranteeing security and reliability of the system, it must adopt the system and products which are cheap and good, economical and practical and increase the cost performance. Thus it brings higher demand for the reliability and robustness of software.

6) System should possess good openness, with clear and standard external interface: Students quality evaluation system is connected with the internet, where various hardware units are not all consistent with standards, so it requires the system to be open, thus network interconnection and interconnection with other devices and systems can be achieved.

7) System data backup and incident record: They include the following two parts:

a) Data backup and data recovering: They are the important link of network database. Harassment and attack form hacker are inevitable during the operation of database. So, how to keep database steady as usual when it is attacked and how to recover database rapidly and keep it integrated after being attacked.

b) Users' incident record: With many people using the system at the same time, in order to log the incident record when user logging in students' information management system, the software designs event recorder to record time when user managing important events, computer IP and incident content. The event recorder greatly facilitates users' events query and prevents disabled user form logging in.

\section{Design Principle of System}

System design is the most important link to transform demands into software system. The design of system is fundamentally decisive in the quality of software system. System design concludes organization and technology: from the angle of technology, system design must make enough need investigation and acquire detailed information of operating mechanisms, information flow, available data state and users' demand. Then form the 
angle of system engineering, make detailed design of system form top to bottom. According to the software engineering requirements and system characteristics, design principle is fixed as follows:

1) Principle of standardization: System design should attach importance to standardization from the beginning. Specify it from data standardization to management standardization, from data classification, definition to data coding, from technology flow to operation flow.

2) Principle of categoricalness: Insure categoricalness of data on the basis of detailed users' demand analysis to make sure that database information and system functional module can meet the need of users' everyday task.

3) Principle of extensiveness: Establish open system to meet the change of users' demand. Keep space for expansion in data organization, system function and system structure for users' future expansion.

4) Principle of applicability: The system interface is friendly and easy which is convenient for maintaining and updating data.

\section{Architecture Design}

Architecture is the most essential part in software system. This system adopts B/S structure which is a thin client-based architecture with three layers, i.e. database system, application server and client browser.

The first layer-client machine is the interface of client machine and the whole system. Application program of client side is predigested to a general purpose browser. Here the web page has certain interactive function which allows user to input information in the form provided by it and submit it to the background-Web server in the second layer, and ask for processing.

WEB server in the second layer will startup corresponding tenor which responds to browser's requests and dynamically generate and feedback code embedded in processing result to the browser of client machine. If data access is one of requests by client machine, WEB server will complete this task cooperated with database server.

Database server-third layer is to harmonize SQL requests from different WEB server and manage database.

\section{Function Design of the System}

\subsection{Overall Function Design of the System}

Based on survey and analysis of the system, design each function of the students' quality evaluation system in an integrated way, and describe the system function, operating procedure and specifications in separate module. Each function module is operated under the control of main control module. The system function structure of the software is shown in Fig. 1.

\subsection{Function Module design of the System}

The system is designed to achieve systematization, standardization and automatization of all kinds of information, which is the basis of the complication of system function analysis. After gaining accurate user information through timely survey and interview by the developer, function and operations of the system are refined concretely and comprehensively. The main function module needs to be finished by the students' quality evaluation system are as follows.

1) System maintenance and management module: System administrator maintains normal operation of the system, manages the authorization of all the users and prevents illegal amendment, deletion of the information by users to keep the information stable and safe.

a) Code management: Include the functions of establishment of students' information and class, amendment and deletion of information. 
b) User management: In order to ensure security and confidentiality of the system and data, system management offers integrated management of users and operation authorization, which can add, delete and amend users of the system and sets use authorization for each user.

c) System maintenance: System maintenance function is designed for data processing of the system, including backup and recovery of data base, lay in and led off of data table, backup and deletion of history data.

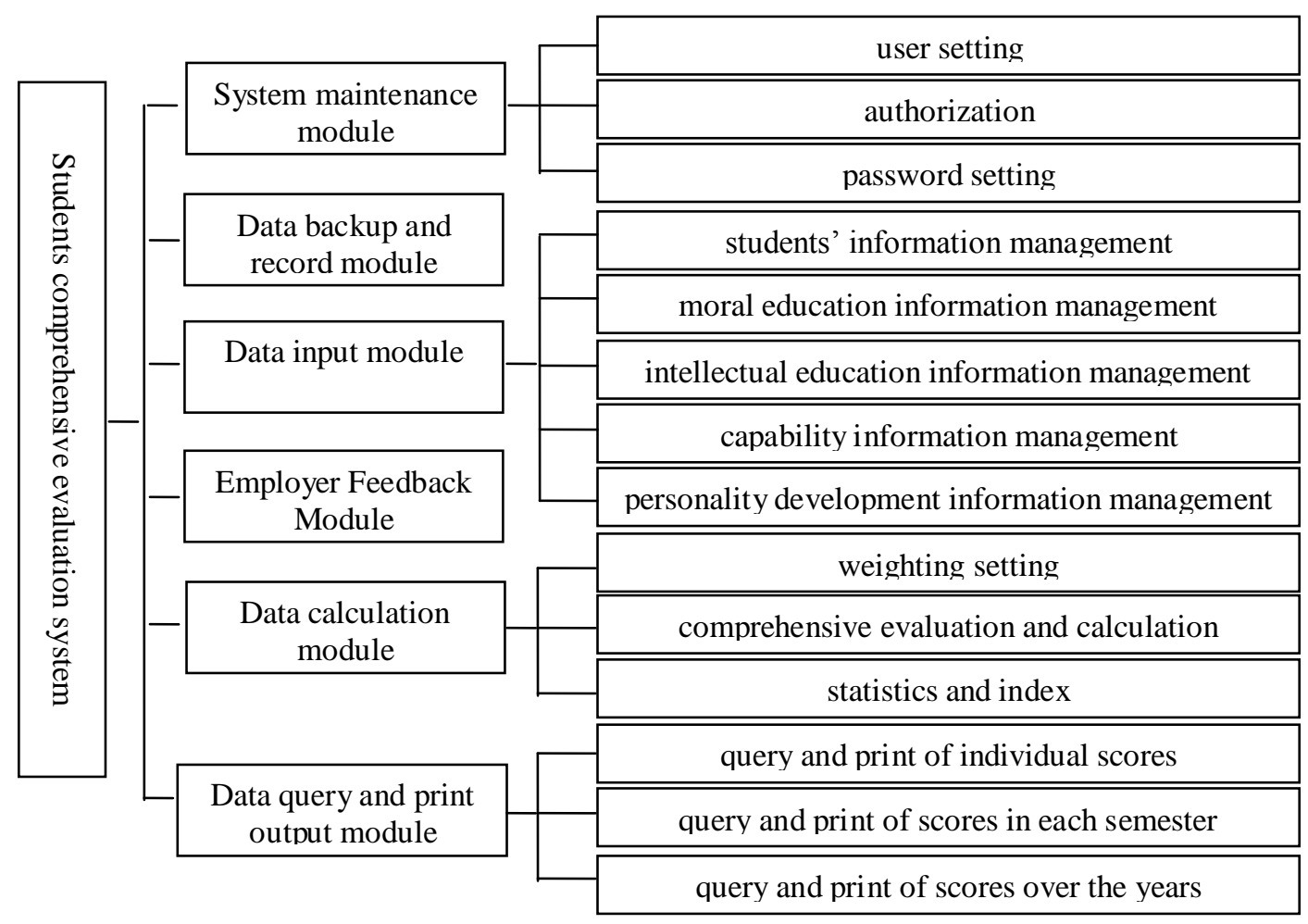

Fig 1. System function structure of the software.

d) Log management: In order to guarantee normal operation of the system, the system record the time when each user logs in and logs out and the specific functions operated by the user at any moment to form computer time log, thus all the operations are recorded and can be found. The administrator can delete contents of the log.

2) Data input module: Data input module consists of the type-in and amendment of the following information, including students' basic information, moral education scores, intellectual education scores, capability scores and personality development scores. Students' basic information include student ID, name, class, sex, date of birth, etc; moral education score, intellectual education score, capability score and personality development score consist of scores of each index separately. Basic information of students should be conversed when they graduate. Backup the information of students who leave the university and delete it from the system.

3) Data calculation module: Calculate the data in accordance with weight coefficient of each index, and then input the data according to the requirements. Calculate the moral education score, intellectual education score, capability score, personality development score and comprehensive evaluation score according to students' information of each aspect. Evaluation results of each aspect, as comprehensive ranking and qualities 
ranking of students, is taken as basis of assessing and granting of the scholarship. Besides, it can be used as standard in recommending proper talents for employing units.

4) Data query and print output module: This module is for data query and print-out of data, including:

- Quality evaluation results of each semester.

- Quality evaluation scores over the years.

- Individual quality evaluation scores of each semester.

- Query according to designated conditions.

- Query of feedback information.

5) Feedback information module: Save feedback information by students and employing units in data base for query and searches.

6) Searches and statistics of students' information: Students' administrator can inquire about students' information through searches. Search methods include the followings:

- Search according to student ID and name

- Search according to student name and class

- Search according to student ID and class

\section{Analysis of System Security Model}

As a defending layer, strengthening of the application program is one indispensable part in any kind of security model. Reinforcing the protection for security of operational system can only provide security of certain extent. The developer is responsible for integrating the security protection into the application program in order to provide special protection for the areas which the application program of architecture has access to. Application program is present in the system environment, so it is impossible to examine security of the application program without considering the whole system. Each application program needs its own security model, but development of the programs should be in accordance with a set of standard guidelines. Basis of any successful security policy of application program is stable identification, authorization means and secure communications to ensure security and integrality of confidential data. Authorization process is responsible for controlling which resources the browsers that have passed the identification have access to and what kind of operations can be carried out by the browsers. The accessible resources include not only files and data base but also system level resources, such as registration table, configuration data. Many programs don't directly authorize the users to have access to the bottom resources, however, it authorizes the operations can be carried out by the browser users by means. This is mainly because that it takes scalability and manageability of application system into consideration.

This application program is multi-user oriented, so different users should be endowed with different authorization, thus security of application program can be guaranteed, advanced level users can have higher authorization and ordinary users only have the operation functions endowed by the administrator.

This application program adopts three-grade user strategy for security management. Users of different grades can use different functions, that is to say, system administrator user has the right to manage and distribute authorization for ordinary and advanced level users; however, the ordinary and advanced level users have no function and right of management. Administrator user has the right to add new users, but ordinary users only have the operational authorization of browse and inquiry; advanced level user has the authorization of decision operation, besides the authorization of ordinary users. They have operation authorization over students' quality evaluation information, moral education scores, intellectual education scores, capability scores and personality development scores. Thus operation authorization of users can be managed effectively, security of application program can be guaranteed effectively, which set a defending layer for security of the system.

In order to prevent the unexceptional failure from destroying data, data backup function is designed in the system, which saves the backup data into text file periodically. If data in the data base is destroyed, use Import function to transfer the text file to data window, and then save it in the data base. 


\section{Conclusions}

Building quality evaluation system enables students management by computer, which is convenient for teachers, students and employing units to inquire about all kinds of information of students on the internet; meanwhile, it has many other functions, including improving work efficiency greatly, saving human resource, enhancing level of management, achieving "paperless" university students management, changing the backwardness of students' quality management and achieving modernization of evaluation.

\section{Acknowledgment}

The research was sponsored by the key scientific and technological project of the Education Department of Henan Province. We would like to thank the referee for his/her careful reading and helpful suggestions.

\section{References}

[1] Frank R.Giordano, Maurice D.Weir, William P.Fox, A First Course in Mathematical Modeling, Thomson, 2001.

[2] Jiang Qiyuan, Mathematical Model(in Chinese), Higher Education Press, 2003.

[3] William F.Lucas, Modules in Applied Mathematics.Vol.3:Discrete and System Models, Springer-Verlag, 1996.

[4] Tang Huanwen, Mathematical Model: An instroduction(in Chinese). Higher Education Press, 2002.

[5] Zhou Wei, Gao Chuan, "Modeling of Students'Comprehensive Assessment System Based on UML(in Chinese)", Computer and Modernization, no.10, pp.147-153, October 2009.

[6] Zhang Yun, "Reform of Comprehensive evaluation index system for University Students(in Chinese)", Journal of South China Normal University(Social Science Edition), no.3, pp. 141-143, June 2009.

[7] He Yongqiang, "Study on the Comprehensive Evaluation System for University Students ,"TEIN2010, 2010.

[8] Huang Tiyun, Management Information System(in Chinese), Higher Education Press, 2000.

[9] Liu Zhenhua, The Design and Implementation of Web-based Integrated Student Management Information System(in Chinese), CHINA EDUCATION INFO, No.12, 2006, pp. 47-48.

[10]Wang Shan, An Introduction to Database Systems(in Chinese), Higher Education Press, 2006.

[11] Paul, Allen, Component-Based Development for Enterprise Systems, Cambridge Universicy Press, 2003. 